\section{KENTRON}

REVUE PLURIDISCIPLINAIR

DUMONDE ANTIQUE

\section{Kentron}

Revue pluridisciplinaire du monde antique

$23 \mid 2007$

La démesure (fin)

\title{
Quand l'ascèse devient péché : les excès dans le monachisme byzantin d'après les témoignages contemporains
}

\section{Vincent Déroche}

\section{(2) OpenEdition}

Journals

Édition électronique

URL : http://journals.openedition.org/kentron/1752

DOI : 10.4000/kentron. 1752

ISSN : 2264-1459

Éditeur

Presses universitaires de Caen

\section{Édition imprimée}

Date de publication : 31 décembre 2007

Pagination : 167-178

ISBN : 978-2-84133-327-9

ISSN : 0765-0590

\section{Référence électronique}

Vincent Déroche, "Quand l'ascèse devient péché : les excès dans le monachisme byzantin d'après les témoignages contemporains », Kentron [En ligne], 23 | 2007, mis en ligne le 16 mars 2018, consulté le 17 novembre 2020. URL : http://journals.openedition.org/kentron/1752 ; DOI : https://doi.org/ 10.4000/kentron. 1752

\section{(c) $(1)(9)$}

Kentron is licensed under a Creative Commons Attribution-NonCommercial-NoDerivatives 3.0 International License. 


\section{QUAND L'ASCÈSE DEVIENT PÉCHÉ: LES EXCĖS DANS LE MONACHISME BYZANTIN D'APRÈS LES TÉMOIGNAGES CONTEMPORAINS}

L'ascèse est par nature le dépassement des limites de la nature humaine au nom de la transcendance: l'ascèse du moine antique le pose comme figure de la mort au monde, et ce porteur de "l'habit angélique» (une des appellations courantes de la tenue monastique) s'affranchit de son corps. Il est commode de partir d'un article bien connu de R. Browning sur un saint qui vécut vers 600 après J.-C., Théodore de Sykéôn ${ }^{1}$ : le jeune Théodore commence son ascèse vers douze ans en s'ensevelissant pour des semaines dans une cavité sous l'autel d'une petite église isolée, d'où on le sort à demi mort; il continue en passant ses nuits à psalmodier et en réduisant sa ration alimentaire à un niveau dérisoire, tout en vivant exposé aux intempéries, enfermé volontaire dans une cage en fer. Il variera ses méthodes d'ascèse au long de sa vie, mais le principe en reste cohérent: l'ascète choisit d'incarner anthropologiquement une forme de contre-humanité, affranchie non seulement de la sexualité, mais encore des besoins physiologiques normaux (faim, sommeil, chaleur, etc.); bien entendu, cet affranchissement n'est jamais complet, et les sources hagiographiques l'exagèrent, mais la tendance est indubitable. Autre figure cohérente de l'ascèse, l'ermite «brouteur» (boskos) des steppes de Syrie-Palestine qui (à l'imitation de Jean-Baptiste) ne se nourrit que d'herbes sauvages mangées crues, et vit errant sans toit avec des "vêtements » de feuilles et de végétaux, ou même totalement nu : triomphe du cru sur le cuit, du retour à l'état de nature sur la civilisation urbaine antique, le boskos s'oppose aussi radicalement à l'humanité normale en revivant la condition d'Adam au Paradis terrestre avant la Chute ${ }^{2}$. De nos jours, ceux qui seraient témoins passifs d'une telle ascèse encourraient certainement l'accusation de non-assistance à personne en danger. Pour les contemporains, du moins ceux qui étaient chrétiens, l'approbation courante de ces pratiques reposait bien entendu sur une conception de la grâce divine comme intervention quotidienne à laquelle pouvaient se remettre ces saints hommes, qui suivaient ainsi à la lettre des préceptes

1. Browning 1981.

2. Flusin 1983; Patlagean 1968. 
évangéliques comme "Le Fils de l'homme n'a pas où reposer sa tête ». L'ascèse étant par nature dépassement de la norme humaine, pouvait-il y avoir une mesure de l'ascèse, et donc un risque de démesure? L'excès comportait le risque suprême du suicide, péché grave par excellence, puisqu'il retire à Dieu son pouvoir de fixer les termes de la vie.

Or, l'idée d'une mesure souhaitable de l'ascèse apparait très vite dans l'histoire du monachisme, et qui plus est dans des sources liées aux milieux monastiques et non chez des opposants éventuels. Rappelons les données habituellement reçues pour le monachisme: prémisses au $\mathrm{III}^{\mathrm{e}}$ siècle, véritable essor très rapide dès le début du IV siècle avec les figures tutélaires d'Antoine et Pachôme en Égypte. Or, ce sont la Vie d'Antoine, rédigée dans le troisième quart du IV siècle, sans doute par Athanase d'Alexandrie, et le Traité pratique d'Évagre le Pontique, rédigé vers la fin du IV $V^{e}$ siècle, qui mettent en garde contre des excès d'ascèse, c'est-à-dire la première biographie d'une pratique monastique et le premier traité théorique du monachisme! La mise en garde contre les excès vient paradoxalement avant l'attestation littéraire de ces excès, mais renvoie certainement à des expériences concrètes dont nous n'avons plus de trace directe. Voici donc les conseils que la Vie d'Antoine prête à son héros:

Souvent, quand nous dormons, ils [les démons] nous réveillent pour la prière. Et cela, ils le font sans interruption, presque au point de ne pas nous permettre de dormir. Quelquefois aussi, se cachant sous les traits de moines, ils feignent de parler comme des hommes pieux, pour nous égarer par leur ressemblance extérieure et pour entraîner ensuite où ils veulent ceux qu'ils ont trompés. Mais il ne faut pas faire attention à eux, même s'ils nous éveillent pour la prière, s'ils nous conseillent de ne pas manger du tout, ou feignent de nous accuser et de nous reprocher des fautes qu'ils savent que nous avons autrefois commises. Car ce n'est pas par piété ou vérité qu'ils font cela, mais pour porter les gens simples au désespoir et leur faire entendre que l'ascèse est inutile, pour donner aux hommes la nausée de la vie solitaire, sous prétexte qu'elle est insupportable et extrêmement lourde, et pour faire obstacle à ceux qui vivent pour les combattre ${ }^{3}$.

Dans la Vie d'Antoine, les démons sont omniprésents, et il est donc logique que toute faute dans l'ascèse leur soit attribuée : l'excès ne serait pas inné à la nature humaine, une potentialité inévitable d'hubris, mais le résultat d'une ruse subtile du démon. Au lieu de tenter directement le moine par le vice, il l'exhorte à une vertu inaccessible, pour que l'échec inévitable l'écarte de la vertu accessible: le moine est comme pris à son propre piège, et on notera la mention explicite de la privation de sommeil et de nourriture. Le Traité pratique s'appuie sur une expérience intime du monachisme, des années aux Kellia dans le désert qui permettent à Évagre d'interpréter

3. Vie d'Antoine, chap. 25, p. 307. 
plus finement les ressorts psychologiques de cette tentation : lorsque le moine commence à maîtriser l'abstinence,

alors, il [le diable] pousse l'intellect à désirer l'ascèse la plus stricte ; à la suite de quoi il lui présente aussi les compagnons de Daniel, leur vie pauvre d'alors et les céréales; il évoque d'autres anachorètes qui ont tout le temps vécu ainsi ou qui ont commencé à le faire, et il le force à les imiter, pour que, en poursuivant une abstinence démesurée, il n'atteigne même pas celle qui est à sa mesure, le corps n'en étant pas capable à cause de sa faiblesse; en réalité, [ce démon] bénit en paroles et maudit dans son cour; il ne convient pas, à mon avis, qu'on lui obéisse ni qu'on s'abstienne de pain, d'huile et d'eau. Car ce régime est excellent, les frères l'ont expérimenté, et cela sans aller jusqu'à la satiété et une fois par jour. Je serais étonné en effet que rassasié de pain et d'eau on puisse recevoir la couronne de l'impassibilité ; j'appelle impassibilité non celle qui empêche les péchés en acte - cela s'appelle abstinence - mais celle qui retranche intérieurement les pensées passionnées, celle que saint Paul a nommée circoncision spirituelle du Juif invisible. Si on est découragé par mes propos, qu'on se souvienne de l'Apôtre, le vase d'élection qui «dans la faim et la soif» a accompli sa course. Le démon d'acédie imite aussi ce démon: il suggère à l'ascète endurci une retraite absolue et l'invite à rivaliser avec Jean Baptiste et avec Antoine, le premier des anachorètes, afin que, ne supportant pas cette retraite prolongée et inhumaine, il s'enfuie honteusement, abandonnant la place : le démon peut alors dire en se vantant: «J'ai prévalu contre lui ${ }^{4}$.

Le mouvement monastique a déjà une histoire et une mythologie propre, avec les grandes figures de l'Ancien Testament, de Jean-Baptiste et d'Antoine (déjà perçu à travers le filtre des apophtegmes et de l'hagiographie), et pour tempérer l'effet de ces mythes mobilisateurs, promesse trompeuse d'un idéal asymptotique, il faut opposer l'expérience des «frères", c'est-à-dire des générations de moines qui se sont déjà succédé. Le régime prôné par Évagre n'a rien de laxiste, puisqu'il est assez austère pour laisser subsister la faim («sans aller jusqu’à la satiété»), mais il écarte les rêves de nourriture purement miraculeuse ou purement naturelle. Cette modération se comprend d'autant mieux que le but d'Évagre n'est pas de battre des records d'ascèse, mais d'arriver à un but proprement spirituel, la clarification de l'âme dans la sérénité en vue de la contemplation; le jeûne, modéré, n'est qu'un moyen pour un des degrés de perfection à atteindre. L'autre versant de ces conseils de prudence nous est fourni par les récits d'échecs dans l'ascèse, plus tardifs. Le célèbre hagiographe Cyrille de Scythopolis, au VI siècle, a dû lui-même interrompre une ascèse trop rigoureuse pour ses capacités et en revenir au mode cénobitique, plus indulgent ${ }^{5}$. Même dans son grand cénobion, Théodose le cénobiarque a dû aménager, à

4. Évagre, Traité pratique, chap. 35, p. 275-277.

5. Flusin 1983, 30 . 
la même époque, un espace pour accueillir les moines frappés de maladies mentales plus que physiques après des excès d'ascèse ${ }^{6}$.

La pratique poussée de l'ascèse dans un milieu chrétien rencontre une autre contradiction interne inévitable : l'ascète qui recherche la perfection personnelle se voit contraint par le devoir d'humilité de la nier devant autrui, et contraint de l'afficher par le devoir d'édification d'autrui. Ce dilemme entre exhibitionnisme et humilité est souvent mis en scène dans les sources les plus anciennes sur le monachisme égyptien, les Apophtegmes, et dans l'hagiographie, dès la Vie d'Antoine qui fait fuir son héros toujours plus loin du monde habité, sans réussir à dissuader visiteurs et disciples. D'un côté, le moine vise par modestie à être un «serviteur caché » (kruptos doulos), de l'autre il faut que ce serviteur soit révélé pour la plus grande gloire de Dieu - d'où la solution ingénieuse de plusieurs histoires édifiantes qui font éclater la sainteté du héros juste à sa mort, après une vie incognito ${ }^{7}$. L'ascèse exhibée trouve sa forme la plus accomplie dans le stylitisme, où l'ascète passe sa vie au sommet d'une colonne, exposé aux rigueurs des intempéries, mais aussi à tous les regards, avec des colonnes de plus en plus hautes pour marquer la progression dans la performance ${ }^{8}$. C'est ainsi que les visiteurs peuvent compter le nombre effarant des proskynèses auxquelles se livre quotidiennement Syméon Stylite l'Ancien ${ }^{9}$, et on a pu comparer l'évidente concurrence entre ascètes pour l'attention du public à celle que se livrent aujourd'hui les champions sportifs. En effet, malgré l'individualisme extrême de la prouesse ascétique, l'ascèse est aussi un phénomène collectif qui réunit un spécialiste et son public: au-delà de ses déterminations psychologiques propres, le « virtuose de la sainteté » (Max Weber) est comme le délégué d'une communauté. Ce groupe humain est une réalité très concrète dans l'esprit de ses membres, les dévots d'un ascète, parce qu'ils attendent de celui-ci les pouvoirs d'intercession en leur faveur (guérison, prédiction, secours divers) que ses efforts ascétiques sont censés lui conférer ${ }^{10}$. Il y a donc de forts enjeux sociaux, comme le révèle l'histoire de l'anachorète endurci qui ne supporte pas la nouvelle concurrence d'un collègue qu'il traite d'imposteur (epithetès), parce que les laïcs vont vers le nouveau venu ${ }^{11}$.

Mais cette comparaison inévitable entre ascètes crée des tensions surtout avec une autre catégorie de "spécialistes » moins brillants à première vue, les cénobites : ceux-ci vivent dans un bâtiment commun, un monastère (koinobion, une «vie

\footnotetext{
6. Canivet 1962.

7. Voir Flusin 2004.

8. Trois successives pour Syméon Stylite le Jeune.

9. Un jour, un visiteur perd le compte une fois arrivé à 1244, mais Syméon continue : Théodoret, Histoire Philothée, chap. 26, p. 204-205.

10. Voir supra note 1.

11. Clugnet 1905, 41-42.
} 
commune ») sous l'autorité d'un abbé qui est leur responsable. L'obéissance et l'esprit communautaire sont les vertus essentielles du cénobite, et elles s'accommodent mal de la surenchère individualiste de la prouesse ascétique. La figure de Syméon Stylite l'Ancien fournit le cas par excellence de cette tension qui amène le jeune moine à quitter le koinobion pour pratiquer sa nouvelle ascèse en solitaire; le dossier philologique est malheureusement complexe, puisque sa vie nous est rapportée par deux sources en grec (une notice de Théodoret dans son Histoire Philothée - encore du vivant de Syméon! - et une Vie par un certain Antoine ${ }^{12}$ ), et plusieurs Vies en syriaque, encore inédites pour certaines, mais apparemment liées malgré des variantes considérables ${ }^{13}$; les perspectives de ces sources sont trop diverses pour qu'on puisse reconstituer une histoire continue en les juxtaposant ${ }^{14}$, et nous nous contenterons de présenter des passages de la Vie syriaque ${ }^{15}$ et de Théodoret ${ }^{16}$. Dans le texte syriaque, Syméon, dans ses débuts au monastère, apparaît comme l'homme de l'ascèse d'exception, qui s'enterre par exemple jusqu'à mi-poitrine dans le jardin du monastère tout l'été, pour se mortifier par la chaleur, ou jeûne deux ou trois semaines de suite; la crise vient des autres moines, qui (mus par l'envie, à en croire l'hagiographe) se coalisent pour venir se plaindre à l'abbé avec ce grief caractéristique: "S'il ne reste pas au même niveau que nous, il ne peut pas rester ici», ou encore: "S'il ne se conforme pas aux frères, qu'il quitte le monastère! ». On en déduit qu'il s'agit d'un régime cénobitique assez souple, bien différent de celui de Pachôme ou plus tard du Stoudios : il n'y a pas de règle détaillée, et les moines sont plutôt juxtaposés qu'encadrés; l'abbé est censé veiller à empêcher les excès par rapport à une norme tacite que rappellent les frères, mais peut autoriser des différences, et semble protéger ici l'exception de Syméon (comme dans l'incident de la corde). La communauté se maintient autour de la liturgie et plus spécialement de l'Eucharistie, et c'est cette dernière qu'on contraint Syméon à prendre après un mois de cachette clandestine, pour marquer son retour à la norme commune ${ }^{17}$. Les bornes sont franchies avec l'incident de la corde que Syméon serre sur sa taille sous ses habits, pour se blesser sans qu'on le voie, et qui le fait repérer au bout d'un moment par la puanteur que dégagent les plaies infectées. Cette première confrontation avec la norme cénobitique tourne donc au conflit insoluble que ne peut plus couvrir l'autorité de l'abbé. Le passage de Théodoret nous présente Syméon un peu plus tard, après sa fuite du monastère et son installation dans son propre ermitage à Tellneshin,

12. Lietzmann 1908; Théodoret, Histoire Philothée, chap. 26, p. 159-215.

13. Voir Doran 1992.

14. Voir Flusin 1993a.

15. Doran 1992, chap. 16-17, p. 112-114.

16. Théodoret, Histoire Philothée, chap. 26, p. 172-176.

17. Le thème de l'ascète dispensé de l'obligation de liturgie et d'Eucharistie est un passage obligé de l'hagiographie et des histoires édifiantes; voir Déroche 2002. 
mais avant de monter sur la colonne, simple reclus. Au bout de trois ans, il se sent assez fort pour avoir « envie à l'exemple de Moïse et Élie, ces hommes divins, de rester quarante jours sans manger ${ }^{18}$, et il demande au périodeute Bassos l'autorisation de passer ces quarante jours sans vivre ni boisson, dans sa pièce avec la porte scellée avec un enduit de boue qui permettra d'authentifier la performance (tous doivent pouvoir vérifier que l'ascète n'a pas «triché », encore un souci évident du public). Le périodeute (litt. "celui qui fait des tournées ») est un délégué de l'évêque pour contrôler dans un secteur de la campagne les clercs et les moines, et notre texte implique qu'il a une autorité de contrôle sur les pratiques ascétiques des ermites de son ressort: il ne consent à permettre l'entreprise qu'à condition d'éviter une forme de suicide, "le plus grand et le premier des crimes", et Syméon place donc dans sa cellule une jarre d'eau et dix pains, pour garantir sa survie. Au bout des quarante jours, Bassos trouve un Syméon presque agonisant, et les pains et la jarre intacts : on ranime l'ermite et on lui fait prendre l'Eucharistie ${ }^{19}$, avant qu'il ne rompe vraiment le jeûne par des plantes sauvages crues, les aliments caractéristiques du boskos. Comme dans le monastère avec l'abbé, Syméon va vers la transgression des limites de l'autorité du périodeute, en mettant sa vie en danger, avec cette différence que le dénouement est heureux: la sainteté du héros est reconnue par " cet admirable homme de Dieu qu'était Bassos », le conflit latent entre charisme et institution est soigneusement désamorcé par Théodoret. L'auteur met ensuite en scène le retour de Bassos dans son propre "troupeau ", autrement dit un koinobion de plus de deux cents moines dont il est l'abbé et à qui il impose une vraie austérité : cénobitisme et érémitisme sont ici des degrés différents d'une même entreprise plutôt que des pratiques antagonistes. Il n'en reste pas moins que les textes évoquent le danger de démesure sans véritablement proposer de solution : un peu comme ceux qui prouvent le mouvement en marchant, Syméon prouve la validité de sa démarche par son succès et non par une argumentation dont on pourrait tirer une règle ; la sainteté ascétique est par nature un cas limite hors normes, réservé à des élus dont on peut seulement discerner les charismes exceptionnels sans pouvoir les réguler. Le même Théodoret insère d'ailleurs dans son récit sur Syméon un épisode analogue avec le chorévêque ${ }^{20}$ Mélèce, mais avec un dénouement différent: Mélèce persuade Syméon de renoncer à porter une chaîne de fer au pied

18. Exode 24, 18; III Rois, 19, 8; mais le vrai modèle, qui n'est pas cité pour ne pas susciter le soupçon de vaine gloire, est bien entendu le jeûne de Jésus de quarante jours au désert avant sa vie publique (Matthieu 4, 1-2; Luc 4, 1-2). On attendrait que Syméon choisisse le temps du Carême pour cette entreprise, mais le texte ne le dit pas explicitement.

19. Voir supra note 17.

20. Une sorte d'évêque auxiliaire pour la campagne (chôra) dans les diocèses très étendus. 
puisque la conscience suffit à imposer au corps des liens spirituels, il [Syméon] céda, et accueillant docilement le conseil, il fit venir un forgeron et lui ordonna d'ouvrir la chaîne $e^{21}$.

Syméon Stylite le Jeune, dans sa longue carrière de la seconde moitié du VI siècle, a certainement imité Syméon l'Ancien, et la ressemblance a pu être renforcée par son hagiographe ${ }^{22}$. Nous retrouvons donc les conseils de modération donnés par le stylite âgé qui assure sa formation ${ }^{23}$, des manifestations de franche haine de la part des autres moines du monastère ${ }^{24}$, le même épisode de la corde enfoncée dans les chairs qui lui attire des reproches de l'ancien ${ }^{25}$, et enfin l'étonnement désespéré de l'ancien devant une conduite qu'il assimile à une tentative de suicide ${ }^{26}$. La prédiction était trop pessimiste, puisque Syméon a vécu plus de soixante ans sur sa colonne, mais elle montre bien l'enjeu de cette recherche permanente des limites.

On considère habituellement qu'après les « virtuoses de l'ascèse » de l'Antiquité tardive, le monachisme byzantin médiéval prend une tournure moins extrême : plus intégré à la société, à l'économie et aux réseaux familiaux, il est beaucoup moins un envers de l'humanité «normale». La tension entre les différentes variantes du monachisme persiste néanmoins ${ }^{27}$, et se reflète dans la question des degrés d'ascèse souhaitables ou admissibles, comme le montrent les démêlés de Pierre d'Atroa. Ce moine, bien représentatif du monachisme bithynien de l'époque, oscillant entre cénobitisme et érémitisme, reste assez proche de l'ascèse ancienne sur les contreforts de l'Olympe de Bithynie dans les premières décennies du IX siècle ; iconodoule sous l'iconoclasme, il est encore plus indépendant que les stylites précédents de la hiérarchie épiscopale, puisqu'il ne reconnait pas comme évêques ceux qui sont pour lui des hérétiques. Mais c'est dans le milieu plus informel des iconodoules qu'il va néanmoins rencontrer des accusateurs, moines et évêques en exil qui contestent les miracles de Pierre et prétendent qu'ils sont dus au démon (selon son hagiographe Sabas, c'est bien entendu par jalousie devant les miracles dont ils ont été incapables que ces accusateurs agissent) ; Pierre est qualifié de goètos ${ }^{28}$, donc à la fois d'imposteur et de sorcier, et nous retrouvons là l'hésitation bien connue des Byzantins devant leurs saints hommes qui paraissaient souvent dangereusement analogues à leurs concurrents, sorciers, devins et guérisseurs ${ }^{29}$. Pour rétablir sa réputation,

21. Théodoret, Histoire Philothée, chap. 26, p. 180.

22. Pour la date et la rédaction, voir Déroche 1996.

23. Vie de Syméon Stylite le Jeune, chap. 17, p. 14-15.

24. Ibid., chap. 23, p. 19.

25. Ibid., chap. 26, p. 22 ; c'est l'épisode qui relève le plus de l'imitation hagiographique.

26. Ibid., chap. 30, p. 30.

27. Voir Flusin 1993b.

28. Vie de Pierre d'Atroa, chap. 37-38, p. 144-149.

29. Voir Dagron 1992. 
Pierre n'a pas d'autre solution que de se soumettre au jugement de Théodore Stoudite, célèbre higoumène iconodoule en exil, et les Vies de Théodore Stoudite confirment le fait ${ }^{30}$. Or, paradoxalement l'examen de Théodore va porter non tant sur la foi de Pierre que sur ses pratiques ascétiques comme si c'étaient ces dernières qui étaient visées: Pierre explique à contrecœur

[que] c'est la dix-huitième année que je m'abstiens de pain, de vin, de fromage et d'huile, et que je me contente uniquement de légumes et de lentilles, en jeûnant quarante jours ${ }^{31}$, mais il m'arrive aussi de me priver souvent par pénitence de l'eau ellemême depuis les saintes Théophanies jusqu'à Pâques ${ }^{32}$; je n'ai qu'une tunique; je porte du fer ${ }^{33}$ et vais sans chaussures.

Théodore accepte le credo sans réticence, mais oblige Pierre à prendre un vrai repas comme lui et lui impose sandales et manteau avant de se porter garant de Pierre devant tous les iconodoules.

Le choix de Théodore comme arbitre n'est pas innocent: c'est la figure de proue d'un nouveau cénobitisme au Stoudios de Constantinople, qui insiste sur le travail, des austérités modérées, et surtout la soumission à la communauté et à l'abbé; l'obéissance du cénobite implique qu'on n'est pas à soi-même sa propre norme. Par contraste, Pierre, sans être un boskos à l'ancienne puisqu'il accepte les aliments cuits et les vêtements (mais dans l'hiver bithynien, on peut difficilement faire comme dans la steppe syrienne), est néanmoins du côté de l'ascèse solitaire extrême et quelque peu ostentatoire. L'éclairage fourni par les Vies de Théodore Stoudite sur l'incident présente des différences révélatrices: d'une part, la renommée exceptionnelle de

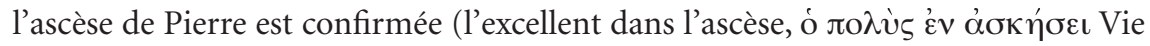
A), d'autre part, le blâme des excès d'ascèse qui reste implicite dans la Vie de Pierre

30. L'étude du RP Laurent aboutit (Vie de Pierre d'Atroa, p. 27-28) à dater l'entrevue en 821; voir Vie de Théodore B, col. 316, et Vie de Théodore C, p. 299 (la version de la Vie de Théodore A est sans intérêt pour ce passage).

31. Pendant le Carême bien sûr; je reproduis avec quelques corrections la traduction du RP Laurent qui, épouvanté par ces excès, a plus ou moins consciemment rectifié le texte en traduisant ce passage comme suit: "pour le jeûne, je me limite au Carême»! C'est au contraire le jeûne total pendant le Carême comme Syméon Stylite, le menu austère de légumes et de lentilles étant le quotidien du reste de l'année.

32. Donc à peu près trois mois par an. Une anecdote célèbre du Pré spirituel au début du VII ${ }^{\mathrm{e}}$ siècle raconte le cas d'un moine qui ne buvait jamais par pénitence: Moschos, Le Pré spirituel, chap. 184, p. 194-195.

33. Il s'agit de chaînes et de plaques de fer cachées sous les vêtements qui représentent un poids considérable, un type d'ascèse bien attesté dès l'Antiquité par exemple par Théodore de Sykéon; Pierre dit ailleurs porter du fer sur tout le corps « comme un condamné »: Vie de Pierre d'Atroa, chap. 12, p. 97. 
d'Atroa devient explicite, en particulier dans la Vie C, qui suggère de refréner «la

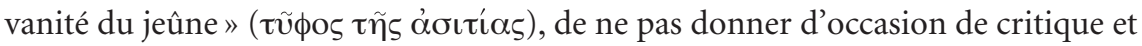

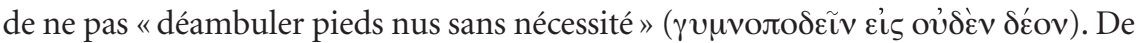
façon discrète, les hagiographes de Théodore, tout en reconnaissant les miracles et la vertu de Pierre, placent l'higoumène stoudite en position de juge du juste degré d'ascèse à défaut d'être grand ascète lui-même. De plus, leurs formulations impliquent que toute ascèse extrême et extravagante entraîne naturellement le soupçon d'imposture; or, c'est bien ce qu'on constate dans une période qui va en gros de la fin de l'iconoclasme à 1204, avec un apogée sous les Comnènes : les milieux cultivés sont suspicieux envers une ascèse de rigueurs physiques, qu'ils considèrent comme celle de parvenus incapables de briller par ailleurs, et s'irritent de la faveur populaire à l'endroit de ces ascètes. P. Magdalino ${ }^{34}$ a bien montré comment cette jalousie a même empêché presque toute "canonisation » de nouveau saint au XII ${ }^{\mathrm{e}}$ siècle; le portrait-charge de ces «saints hommes " populaires que dressent des intellectuels comme Tzetzes en fait des crève-la-faim imposteurs, chargés de fers dont les dévots crédules font des reliques - à peu près l'impression que devait donner Pierre d'Atroa à ses détracteurs. On en trouve l'écho encore dans les hagiographies des IX ${ }^{\mathrm{e}}-\mathrm{XI}^{\mathrm{e}}$ siècles qui mettent en scène des saints non thaumaturges, qui blâment même le recours au miracle comme preuve de sainteté ${ }^{35}$; or, le miracle à Byzance est lié à l'ascèse dont il est la contrepartie positive, et comme pour Pierre d'Atroa, critiquer le miracle revient à critiquer l'ascèse, et vice versa. C'est dans ce contexte qu'il faut apprécier un dernier exemple, la figure attachante de Cyrille le Philéote. Né vers 1015, ce paysan aisé et marié du bourg de Philéa, à un bon jour de marche de la capitale, devient peu à peu ermite sur ses propres biens, suivant des pratiques d'austérité traditionnelles de son propre chef; au bout d'un moment, il s'inquiète du bien-fondé de son ascèse, car il «évitait de s'en tenir à sa propre opinion », et va donc voir un moine réputé de Constantinople, Hilarion, à qui il expose son mode de vie, comme jadis Pierre d'Atroa à Théodore Stoudite. Hilarion approuve tout... sauf le port de fers, car les «bardés de fer» (sidèrophoroi) ne peuvent garder indéfiniment leur ascèse secrète et s'exposent ainsi au danger de la vaine gloire, chercher à plaire aux hommes et non à Dieu. Cyrille demande aussitôt l'autorisation d'enlever ses fers avec ces mots: "Je hais les souffrances qui m'occasionnent un dommage spirituel ${ }^{36}$. Cyrille est à la fois l'égal et l'envers de Pierre d'Atroa: dans une situation analogue, il devance la critique en allant voir une autorité qui lui donne la norme acceptable. Or, sa biographie présente deux personnages de moines qui en font trop

\footnotetext{
34. Magdalino 1981.

35. Flusin 1993b.

36. Vie de Cyrille le Philéote, chap. 16, p. 88-9o et trad. p. 311-313.
} 
et tombent du coup dans le péché: l'hagiographe est soucieux de distinguer son héros des excès connus de son temps ${ }^{37}$.

Danger de la démesure? Le terme même d'hubris est quasi absent de nos textes pour désigner l'excès dans l'ascèse : hubris et les mots de sa famille sont alors réservés aux outrages entre humains, alors que la démesure face à Dieu reçoit des qualificatifs comme vanité (tuphos) ou vaine gloire (kenodoxia), sans doute parce que l'idée d'un vrai défi d'un mortel à un Dieu créateur transcendant tout-puissant est absurde dans l'univers chrétien. Le moine dans l'excès est excessif et outrecuidant non par rapport à Dieu, mais par rapport à lui-même, et éventuellement par rapport aux cohumains qui sont le vrai public visé par son défi; l'aveuglement du présomptueux n'est d'ailleurs pas attribué à Dieu ou à la fatalité, mais aux démons omniprésents qui pervertissent ses efforts en le faisant tomber dans l'orgueil. En revanche, ce bref échantillon de cas limites bien connus fait apparaître deux des paradoxes de l'ascèse chrétienne: elle n'a pas de norme interne concevable, puisqu'elle vise à repousser les limites de l'humain jusqu'à friser le risque du suicide, et les normes éventuellement imposées çà et là à titre prudentiel sont démenties par les exceptions triomphantes des grands saints, par définition hors normes; malgré l'apparence, on n'est jamais ascète tout seul, même pendant des années de solitude au fond du désert, mais d'une part dans le cadre de modèles antérieurs déjà éprouvés par d'autres, quitte à les redéfinir et radicaliser, et d'autre part à destination d'autrui, soit par l'intercession qui est un devoir de l'ascète, soit par l'édification du public par l'exemple et le miracle, même s'ils ne sont connus qu'à la mort de l'ascète. Celui-ci se définit toujours par rapport à la norme d'un groupe social, par rapport à laquelle il doit poser un envers, sa contre-norme, tout en laissant cette dernière dans les limites de l'acceptable par son propre corps et par le corps social tout entier.

Vincent Déroche

CNRS Paris

37. Vie de Cyrille le Philéote, chap. 24, p. 112-117 et trad. p. 337-343 (contre les moines errants, qui ressemblent fort à ceux dénoncés au XII ${ }^{\mathrm{e}}$ siècle), et chap. 42, p. 200 et trad. p. 425 (un moine qui, égaré par le diable, voulait vivre sans même mendier). 


\section{Références bibliographiques}

Browning R. (1981), "The "Low-Level” Saint's Life in the Early Byzantine World», in The Byzantine Saint (14th symposium of Byzantine Studies), S. Hackel (éd.), Londres, University of Birmingham, p. 117-127.

Canivet P. (1962), «Erreurs de spiritualité et troubles psychiques », RSR, 50, p. 161-205.

Clugnet L. (1905), "Vies et récits d'anachorètes », Revue de l'Orient chrétien, 10, p. 39-56.

Dagron G. (1992), «L'ombre d'un doute : l'hagiographie en question », Dumbarton Oaks Papers, 46, p. 59-68.

Déroche V. (1996), "Quelques interrogations à propos de la Vie de Syméon Stylite le Jeune», Eranos, 94, p. 65-83.

Déroche V. (2002), «Représentations de l'Eucharistie dans la haute époque byzantine », Travaux et Mémoires, 14, p. 167-180.

Doran R. (1992), The Lives of Symeon Stylites, Kalamazoo, Cistercian Publications (Cistercian Studies Series; 112).

Évagre (Guillaumont 1971), Traité pratique (ou Le Moine), C. et A. Guillaumont (éd. et trad.), Paris, Éditions du Cerf (Sources chrétiennes ; 170-171).

Flusin B. (1983), Miracle et histoire dans l'ouvre de Cyrille de Scythopolis, Paris, Institut d'études augustiniennes (Études augustiniennes).

Flusin B. (1993a), «Syméon et les philologues, ou la mort du stylite », in Les Saints et leur sanctuaire à Byzance, C. Jolivet et al. (éd.), Paris, Publications de la Sorbonne (Byzantina Sorbonensia; 11), p. 1-23.

Flusin B. (1993b), «L'hagiographie monastique à Byzance au $\mathrm{IX}^{\mathrm{e}}$ et au $\mathrm{X}^{\mathrm{e}}$ siècle. Modèles anciens et tendances contemporaines ", Revue bénédictine, 103, p. 31-50.

Flusin B. (2004), "Le serviteur caché ou le saint sans existence », in Les Vies des saints à Byzance: genre littéraire ou biographie historique?, P. Odorico (éd.), Paris, École des Hautes Études (Dossiers byzantins ; 4), p. 59-71.

KaPlan M. (1997), "In search of Cyril's Philea», in Work and Worship at the Theotokos Evergetis, Belfast, Belfast Byzantine Enterprises (Belfast Byzantine Texts and Translations), p. 213-221.

Magdalino P. (1981), "The Byzantine Holy Man in 12th century», in The Byzantine Saint (14th symposium of Byzantine Studies), S. Hackel (éd.), Londres, University of Birmingham, p. 139-162.

Moschos Jean (Bouchet 2006), Le Pré spirituel. Fioretti des moines d'Orient, C. Bouchet (trad.), Paris, Migne (Les pères dans la foi ; 94-95).

Patlagean E. (1968), "Ancienne hagiographie et histoire sociale », Annales ESC, 1, p. 106126, réimpr. in E. Patlagean (1981), Structure sociale, famille, chrétienté à Byzance (IV $X I^{e}$ siècle), Londres, Variorum Reprints.

Théodoret (Canivet 1979), Histoire Philothée, P. Canivet (éd. et trad.), Paris, Éditions du Cerf (Sources chrétiennes; 257). 
Vie d'Antoine, G. Bartelink (éd. et trad.) (1994), Paris, Éditions du Cerf (Sources chrétiennes; 400).

Vie de Cyrille le Philéote, É. Sargologos (éd. et trad.) (1964), Bruxelles, Société des bollandistes (Subsidia hagiographica; 39).

Vie de Pierre d'Atroa $=$ La Vie miraculeuse de saint Pierre d'Atroa ( $†$ 837), V. Laurent (éd. et trad.) (1956), Bruxelles, Société des bollandistes (Subsidia hagiographica; 29).

Vie de Syméon Stylite l'Ancien = H. Lietzmann (1908), Das Leben des hlg. Symeon Stylites, Leipzig, J.C. Hinrichs (Texte und Untersuchungen zur Geschichte der altchristlichen Literatur : 3. Reihe; 2, Heft 4).

Vie de Syméon Stylite le Jeune = La Vie ancienne de saint Syméon Stylite le Jeune: 521-592, P. Van den Ven (éd. et trad.) (1962), Bruxelles, Société des bollandistes (Subsidia hagiographica; 32).

Vie de Théodore $\mathrm{B}=$ Vie de Théodore Stoudite B, PG, 99, col. 233-328.

Vie de Théodore C = Vie de Théodore Stoudite C, B. Latyschev (éd.) (1914), Vizantiskij Vremennik, 21, p. 258-304. 